\title{
Studies on Novel Bacterial Translocase I Inhibitors, A-500359s
}

\author{
V. Enhanced Production of Capuramycin and A-500359 A in \\ Streptomyces griseus SANK 60196
}

\author{
Yasunori Muramatsu, Masatoshi Arai, Yoshiharu Sakaida, Yasuyuki Takamatsu, \\ Shunichi Miyakoshi, Masatoshi Inukai
}

Received: June 21, 2006 / Accepted: September 12, 2006

(C) Japan Antibiotics Research Association

\begin{abstract}
Streptomyces griseus SANK 60196 produces the novel nucleoside antibiotics A-500359 A, C, D and capuramycin. Enhanced production of capuramycin and A-500359 A was achieved through a number of medium modifications and a series of single colony isolations. The addition of maltose instead of glucose as the carbon source in a primary medium resulted in a 20 -fold increase in the productivity of capuramycin. Furthermore, the addition of cobalt chloride $\left(\mathrm{CoCl}_{2}\right)$ and yeast extract to the medium containing maltose drastically altered the production ratio of A-500359 A to capuramycin. Thus, the yield of A-500359 A increased up to $600 \mu \mathrm{g} / \mathrm{ml}$ in an optimal medium, while the yield in the primary medium was $1 \mu \mathrm{g} / \mathrm{ml}$.
\end{abstract}

Keywords capuramycin, translocase I, A-500359 A

\section{Introduction}

Since bacterial phospho- $N$-acetylmuramyl-pentapeptidetranslocase (translocase I), which catalyzes the first step in the lipid cycle of peptidoglycan biosynthesis, is essential and specific for bacteria, it is thought to be an attractive target for the development of antimicrobial agents. In the course of our screening for bacterial translocase I

Y. Muramatsu (Corresponding author), M. Arai, Y. Sakaida, Y. Takamatsu, S. Miyakoshi, M. Inukai: Lead Discovery Research Laboratories, Sankyo Co., Ltd., 1-2-58 Hiromachi, Shinagawa-ku, Tokyo 140-8710, Japan, E-mail: yasuno@sankyo.co.jp inhibitors, we found inhibitory activity in the cultured broth of Streptomyces griseus SANK 60196 and isolated the novel capuramycin derivatives A-500359s [1]. One of the compounds, A-500359 A, inhibited the enzyme with an $\mathrm{IC}_{50}$ value of $0.017 \mu \mathrm{M}$ and showed potent antimicrobial activity against Mycobacterium smegmatis ATCC 607 with an MIC value of $6.25 \mu \mathrm{g} / \mathrm{ml}$ [2].

However, the productivity of capuramycin and A-500359 A in the strain needed to be improved in order to obtain sufficient quantities of these compounds as substrates for chemical modification sources. In this paper, we describe the enhanced production of capuramycin and A-500359 A in S. griseus SANK 60196 achieved through modifications to fermentation media and a series of single colony isolations.

\section{Materials and Methods}

\section{Materials}

Medium 1 (PM-1) composed of glucose 2\%, soluble starch $1 \%$, pressed yeast $0.9 \%$, Polypepton (Nihon Seiyaku) $0.5 \%$, meat extract (Kyokuto) 0.5\%, $\mathrm{NaCl} 0.5 \%, \mathrm{CaCO}_{3} 0.3 \%$ and CB-442 (NOF Co., Ltd.) $0.01 \%$ (pH 7.4, before sterilization) was used as a primary medium. We also used Basal medium 1 (BM-1) in which any carbon sources were eliminated from the PM-1 medium. Basal medium 2 (BM-2) consisted of maltose $3 \%, \mathrm{NaCl} 0.5 \%, \mathrm{CaCO}_{3}$ $0.3 \%$ and CB-442 (NOF Co., Ltd.) $0.01 \%$ (pH 7.4, before sterilization). Primary medium 2 (PM-2) was a modified version in which $0.5 \%$ Polypepton and $0.5 \%$ meat extract 
were added to BM-2. The optimized medium 1 (OM-1), composed of maltose $3 \%$, Polypepton $0.5 \%$, meat extract $0.5 \%$, yeast extract (Difco) $0.5 \%, \mathrm{NaCl} 0.5 \%, \mathrm{CaCO}_{3} 0.3 \%$, $\mathrm{CoCl}_{2} \cdot 6 \mathrm{H}_{2} \mathrm{O} 0.001 \%$ and CB-442 (NOF Co., Ltd.) $0.01 \%$ (pH 7.4, before sterilization) was used for jar fermentation.

\section{Isolation of Spontaneous Variations}

After 7 to 10 days of growth on ISP\#2 medium (Difco) at $23^{\circ} \mathrm{C}$, spores of $S$. griseus SANK 60196 were inoculated onto plates with the same composition. After a 14-day cultivation at $23^{\circ} \mathrm{C}$, at least 50 colonies were picked up and transferred to a solid medium.

\section{Flask Fermentation}

A culture of S. griseus SANK 60196 and the isolated single colonies grown on ISP\#2 medium for 7 to 10 days were used to inoculate 500-ml Erlenmeyer flasks containing $80 \mathrm{ml}$ of PM-1. Four to five days after inoculation, $2.4 \mathrm{ml}$ of the cultured medium was transferred to $500-\mathrm{ml}$ Erlenmeyer flasks containing the same fresh medium and incubated for 7 to 9 days at $23^{\circ} \mathrm{C}$ on a rotary shaker (210 rpm).

\section{Jar Fermentation}

Two loopfuls of spores from a culture of the strain SANK 60196 were inoculated into a 2-liter Erlenmeyer flask containing $500 \mathrm{ml}$ of PM-2 medium. The inoculated flask was incubated on a rotary shaker $(210 \mathrm{rpm})$ at $23^{\circ} \mathrm{C}$ for 5 days. Then a $300-\mathrm{ml}$ aliquot of the culture was transferred into a 30-liter jar fermentor containing 15 liters of OM-1. Fermentation was carried out at $23^{\circ} \mathrm{C}$ for 8 days with an air-flow rate of 15 liters/minute and an agitation rate of 100 to $400 \mathrm{rpm}$.

\section{Determination of Productivity}

The yields of A-500359 A, C, D and capuramycin in each culture were analyzed by HPLC. The harvested broth was centrifuged $(3,000 \mathrm{rpm}, 10$ minutes $)$ and the resulting supernatant $(5 \mathrm{ml})$ was adsorbed on a Seppak plus cartridge C18 (Waters). The cartridge was washed with $2 \mathrm{ml}$ of water and eluted with $2 \mathrm{ml}$ of $20 \%$ aqueous acetonitrile. The eluate was applied to an HPLC column (Senshu Pak ODSH-2151, Senshu Scientific; mobile phase: acetonitrile $0.04 \%$ aqueous trifluoroacetic acid [8:92]; flow rate: $1.0 \mathrm{ml} /$ minute). A-500359 A and capuramycin were eluted at the retention times of 18.7 minutes and 8.1 minutes, respectively.

\section{Results}

\section{Enhanced Production of Capuramycin}

Although S. griseus SANK 60196 produced $5 \mu \mathrm{g} / \mathrm{ml}$ of capuramycin in PM-1, the medium in which we first detected the inhibitory activity, the productivity was insufficient for chemical derivatization. In order to improve the capuramycin production, we examined the effects of glucose, starch, sucrose, maltose, glycerol and lactose as carbon sources. Fig. 1A shows that maltose at 3\% gave the best production levels, while the other carbon sources failed to improve the production.

The effects of various nitrogen sources were examined using BM-2, a modified medium that was free of any nitrogen sources and contained 3\% maltose instead of glucose. As a result, the production of capuramycin in the medium containing meat extract $0.5 \%$ and Polypepton $0.5 \%$ reached $80 \mu \mathrm{g} / \mathrm{ml}$, while a slight enhancement of productivity was observed using either [meat extract $1 \%$ alone], [meat extract $0.5 \%$ and pressed yeast $0.9 \%$ ] or [Polypepton $0.5 \%$ and pressed yeast $0.9 \%$ ] (Fig. 1B). No production of capuramycin was observed with the other nitrogen sources tested in the experiment. Furthermore, the improvement resulting from the addition of meat extract and Polypepton was cancelled out when the concentration of maltose was raised to $5 \%$ (data not shown).

Next, we investigated the effect of cultivation temperature. As the temperature increased, the growth rate and fragmentation of mycelium increased along with rising $\mathrm{pH}$ (data not shown). However, the best production of capuramycin was observed at the lowest temperature, $23^{\circ} \mathrm{C}$ (Fig. 2). No further enhancement of the production was observed below $23^{\circ} \mathrm{C}$ (data not shown).

The production of capuramycin reached $100 \mu \mathrm{g} / \mathrm{ml}$ using the medium (PM-2), consisting of maltose 3\%, Polypepton $0.5 \%$, meat extract $0.5 \%, \mathrm{NaCl} 0.5 \%, \mathrm{CaCO}_{3} 0.3 \%$ and CB-442 (NOF Co., Ltd.) $0.01 \%$ (pH 7.4, before sterilization) at $23^{\circ} \mathrm{C}$, whereas the strain produced $5 \mu \mathrm{g} / \mathrm{ml}$ of the compound in the primary medium (PM-1) at $28^{\circ} \mathrm{C}$.

\section{Single Colony Isolations}

A series of single colony isolations was carried out in order to obtain stable clones for the production of capuramycin. One loopful of each colony obtained by the method described in Materials and Methods was cultured in 100-ml Erlenmeyer flasks, each containing $20 \mathrm{ml}$ of PM-2 medium at $23^{\circ} \mathrm{C}$ for 3 days, and the resulting seed culture $(2.4 \mathrm{ml})$ was transferred into $500-\mathrm{ml}$ Erlenmeyer flasks, each containing $80 \mathrm{ml}$ of the same medium. After 8 days cultivation at $23^{\circ} \mathrm{C}$, the productivity of the capuramycin 


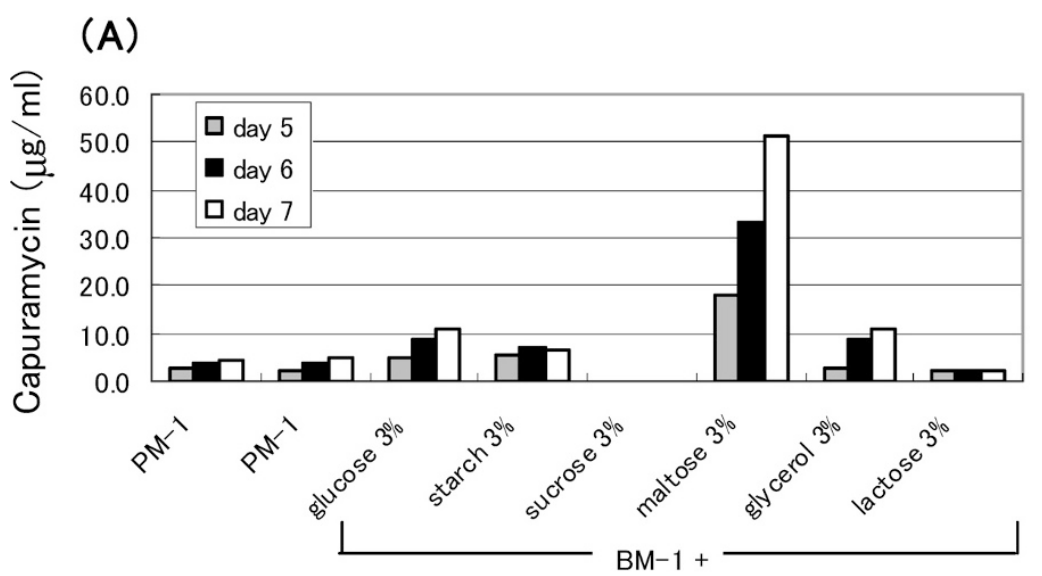

(B)

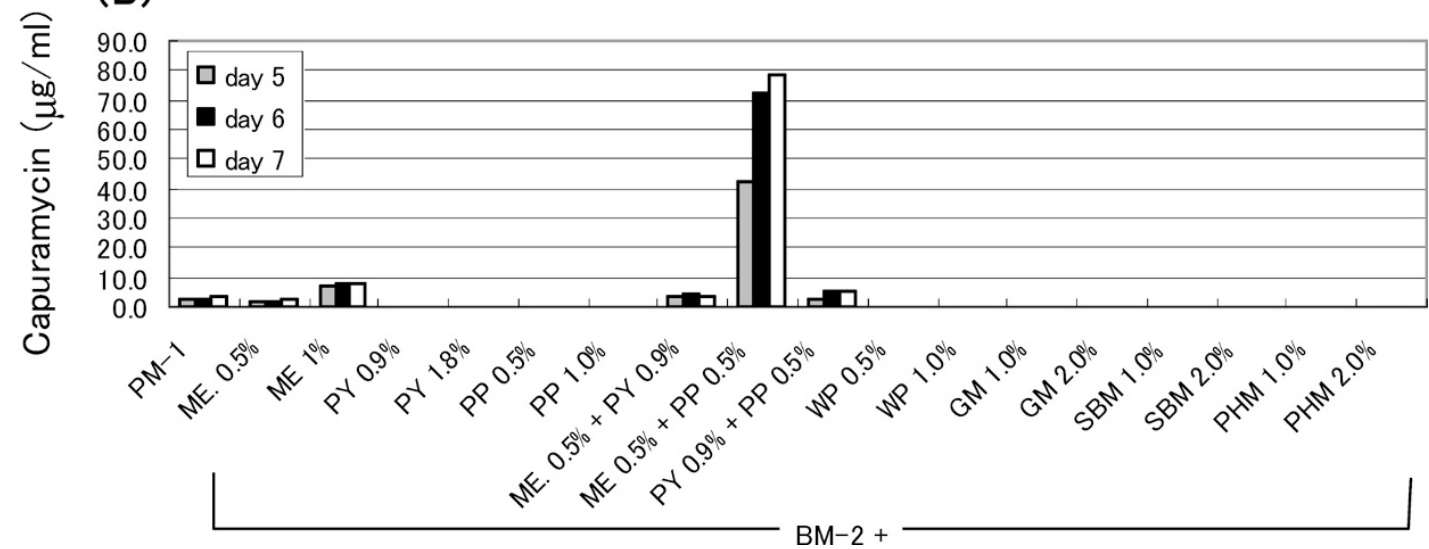

Fig. 1 Effects of various carbon and nitrogen sources on capuramycin production.

(A) Each carbon source shown below was added to BM-1. (B) Each nitrogen source (ME: meat extract, PY: pressed yeast, PP: Polypepton, WP: wheat protein, GM: gluten meal, SBM: soybean meal, PHM: pharmamedia) was added to BM-2 containing $3 \%$ maltose as a carbon source.

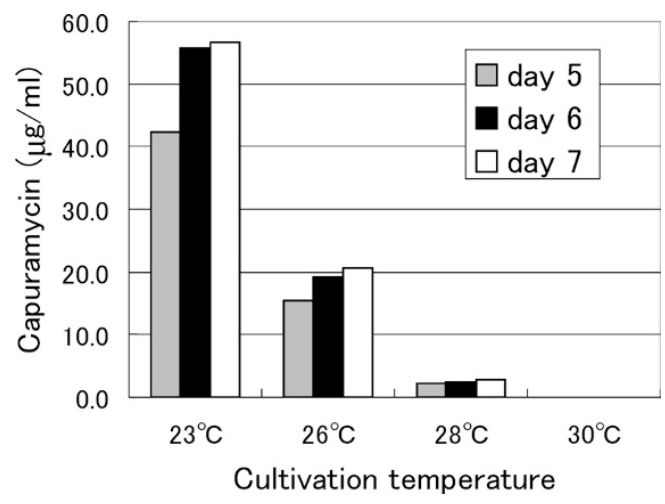

Fig. 2 Effect of cultivation temperature on capuramycin production.

The strain was cultured in PM-1 at various temperatures for 6 to 8 days.

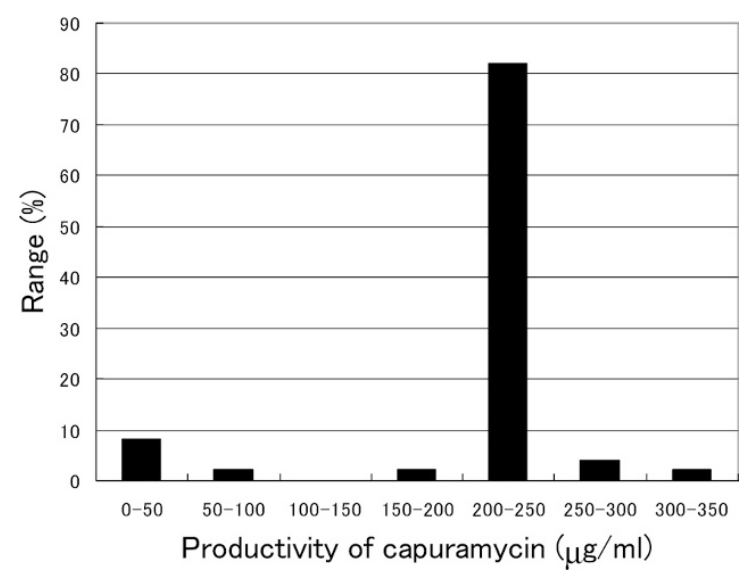

Fig. 3 Production of capuramycin by single colony isolation using strain $1 \mathrm{M}-3$, which showed $247 \mu \mathrm{g} / \mathrm{ml}$ of productivity in the first isolation. 
was determined by HPLC using the method described previously. Two strains showing a productivity of $200 \mu \mathrm{g} / \mathrm{ml}$ or more were obtained in the first isolation. One strain named $1 \mathrm{M}-3$, which showed $247 \mu \mathrm{g} / \mathrm{ml}$ of productivity in the first isolation, was adopted for the second isolation as the parent strain. Ninety percent of the isolates from the second isolation produced over $200 \mu \mathrm{g} / \mathrm{ml}$ of capuramycin.
The most efficacious isolate, designated as $2 \mathrm{M}-30$, produced $319 \mu \mathrm{g} / \mathrm{ml}$ of the compound in the second isolation experiment (Fig. 3).

\section{Enhanced Production of A-500359 A}

Improvement of the production of A-500359 A, the main starting material for derivatization, was needed, since
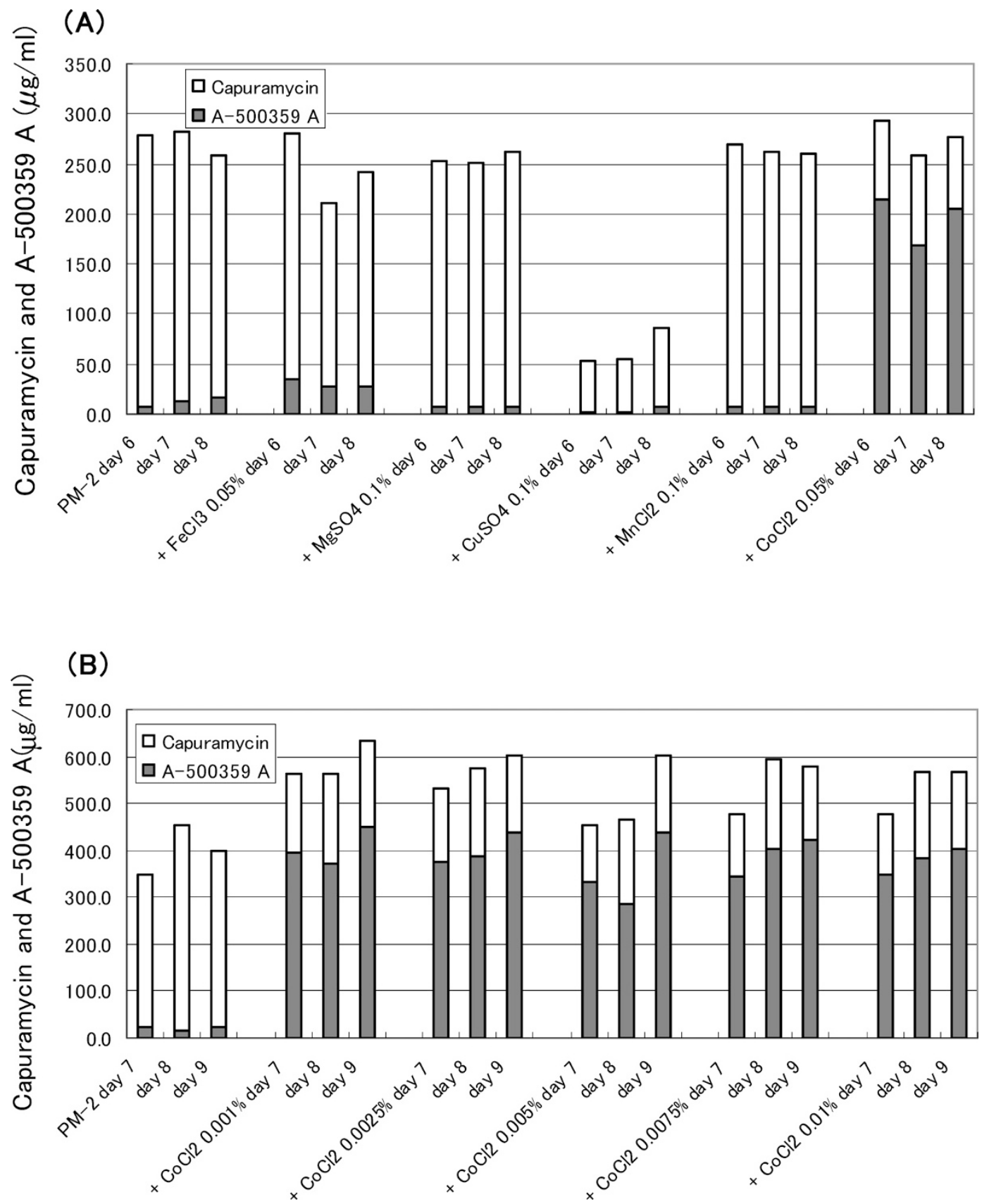

Fig. 4 Effects of various metal ions on the production of capuramycin and A-500359 A.

(A) Each metal ion shown below was added to primary medium-2 (PM-2) described in Materials and Methods. The addition of cobalt chloride drastically altered the production ratio of A-500359 A to capuramycin. (B) Various concentrations of cobalt chloride were added to PM-2 and incubated for 9 days at $23^{\circ} \mathrm{C}$ on a rotary shaker. 


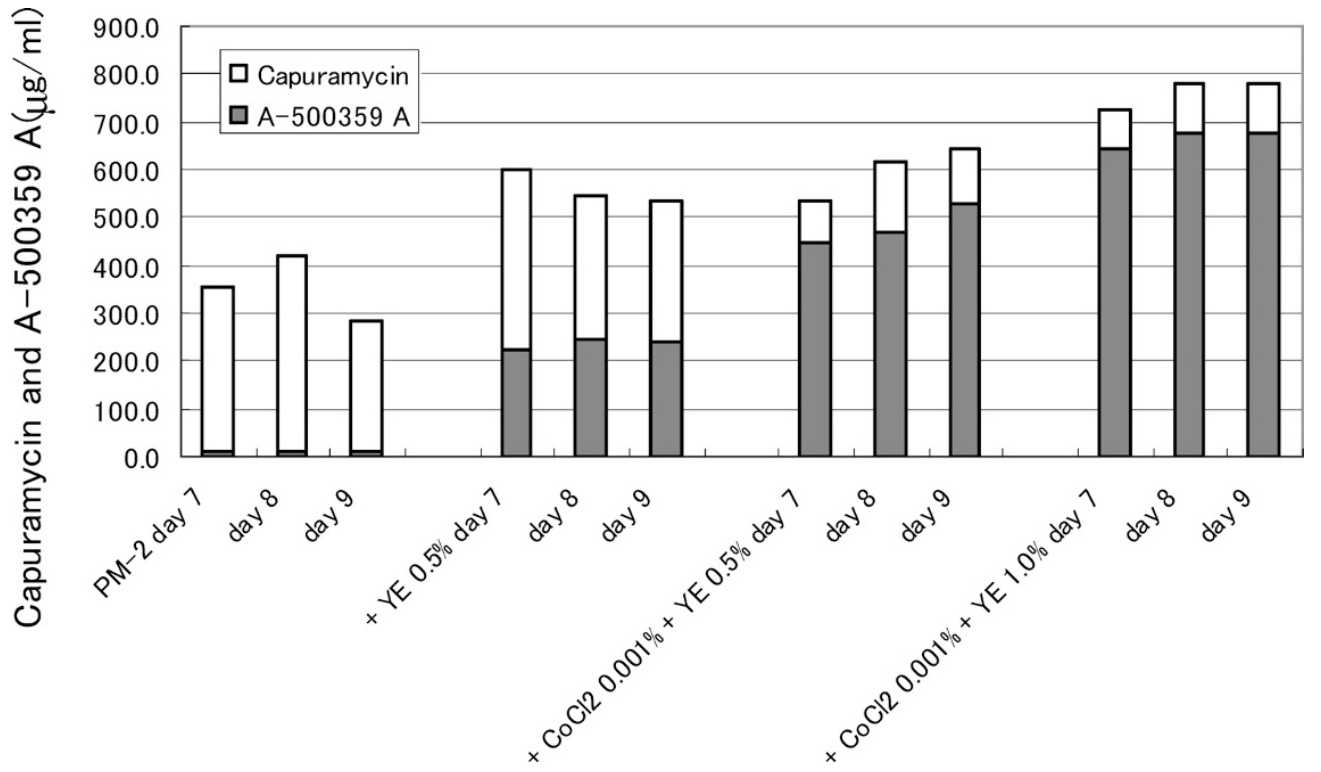

Fig. 5 Effect of cobalt chloride and yeast extract (YE) on the production of capuramycin and A-500359 A.

Each source was added to the primary medium-2 (PM-2) and incubated for 9 days at $23^{\circ} \mathrm{C}$ on a rotary shaker.

A-500359 A was a minor component in the primary medium at the time we first discovered the inhibitory activity. A-500359 A has an extra methyl carbon at the $6^{\prime \prime \prime}$ position on the aminocaprolactam ring of capuramycin and transmethylation might participate in the biosynthesis of A-500359 A. It is known that methylcobalamin, a derivative of vitamin B12, plays a role as a methyl donor in both mammals and bacteria $[3,4]$, so we investigated the effect of various metal ions including $\mathrm{Co}^{2+}$ in order to alter the productivity of A-500359 A. As we expected, the production ratio of A-500359 A to capuramycin changed remarkably when $\mathrm{CoCl}_{2}$ was added to $\mathrm{PM}-2$ medium, while no enhancement effect of A-500359 A production was observed with other ions (Fig. 4A). Next we examined the concentration of $\mathrm{CoCl}_{2}(0.001 \% \sim 0.01 \%)$, and found that $0.001 \%$ of $\mathrm{CoCl}_{2}$ was sufficient to alter the production ratio (Fig. 4B).

Through various supplementation trials, we discovered that an addition of yeast extract enhanced A-500359 A production, and that a synergistic effect was observed when $\mathrm{CoCl}_{2}$ and yeast extract were used together. (Fig. 5) Using the medium containing $\mathrm{CoCl}_{2} \quad 0.001 \%$ and yeast extract $1.0 \%$, the production level of A-500359 A reached $600 \mu \mathrm{g} / \mathrm{ml}$ while the production in the primary medium (PM-1) at the very beginning of the study had been $1 \mu \mathrm{g} / \mathrm{ml}$.

Based on the above investigations, we tried to obtain large amounts of A-500359 A by jar fermentation (Fig. 6). Production of A-500359 A and capuramycin reached

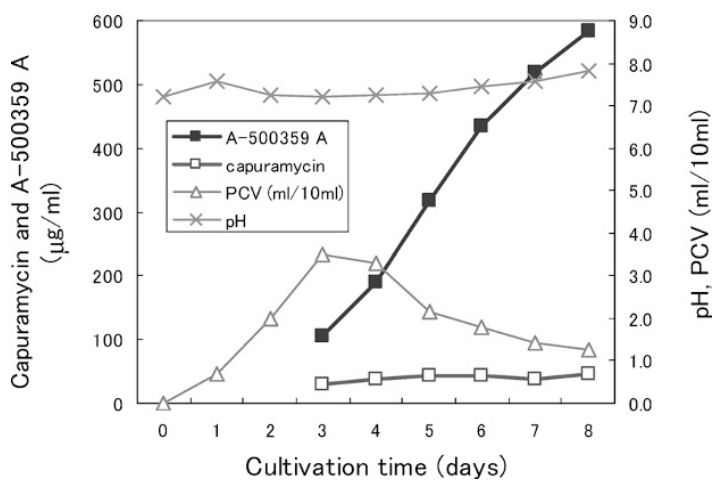

Fig. 6 Fermentative productivity of A-500359 A by $S$. griseus SANK60196 using optimized medium OM-1 in a 30liter fermentor.

* PCV indicates packed cell volume.

$583 \mu \mathrm{g} / \mathrm{ml}$ and $45 \mu \mathrm{g} / \mathrm{ml}$, respectively, in the optimal medium-1 (OM-1) which contained both yeast extract $0.5 \%$ and $\mathrm{CoCl}_{2}$ 0.001\%.

\section{Discussion}

Capuramycin is a nucleoside antibiotic first reported by $\mathrm{H}$. Yamaguchi et al. in 1986 [5]. According to the report, $0.2 \mathrm{~g}$ of capuramycin was obtained using 5 liters of medium composed of glycerol, meat extract, Polypepton and 
$\mathrm{NaCl}$. The productivity in the report was estimated to be $60 \sim 80 \mu \mathrm{g} / \mathrm{ml}$, which was over ten-fold higher than the productivity at the time when we first started this study. Through medium modification using maltose, Polypepton and meat extract, the production of capuramycin could be improved up to $100 \mu \mathrm{g} / \mathrm{ml}$. The desirable effect caused by the addition of meat extract and Polypepton was cancelled out when the concentration of maltose was raised to $5 \%$. This might indicate that the productivity of capuramycin depends on nutrient-limited conditions that are determined by the concentrations of meat extract and Polypepton. Monitoring the culture conditions, we observed that the easily digested nutrients such as glucose were consumed at an early stage of the cultivation, accompanied by a rapid increase of $\mathrm{pH}$, which caused lower productivity. A cultivation temperature of $23^{\circ} \mathrm{C}$ was found to be the most suitable for capuramycin production and exhibited a delayed increase of $\mathrm{pH}$. These observations might indicate that relatively high productivity is achieved when the metabolic turnover of the strain is down regulated to avoid the rapid increase of $\mathrm{pH}$.

When we examined the effect of metal ions added to PM-2 medium as a basal medium, only the addition of $\mathrm{Co}^{2+}$ was effective to alter the main product. The structure of A-500359 A was elucidated as 6"'--methyl derivative of capuramycin, as reported previously [1]. Therefore, a methyl transfer step or steps may be involved in the biosynthesis of A-500359 A. Since methylcobalamin, a derivative of vitamin B12, is known as a methyl donor in both mammals and bacteria [3,4], and it needs $\mathrm{Co}^{2+}$ for its activity, it might play a role in the biosynthesis of A-500359 A in the producing strain. Furthermore, we discovered that the addition of yeast extract enhanced A-
500359 A production, and a synergistic effect was observed when $\mathrm{CoCl}_{2}$ was added together with yeast extract. As yeast extract is known to be rich in vitamins, it might enhance the methyl transfer step(s) in the biosynthesis of A-500359 A via methylcobalamin system(s).

Improvement of the culture conditions resulted in high and selective production of A-500359 A. However, higher productivity may be achieved by further optimization along these lines.

\section{References}

1. Muramatsu Y, Muramatsu A, Ohnuki T, Ishii MM, Kizuka M, Enokita R, Tsutsumi S, Arai M, Ogawa Y, Suzuki T, Takatsu T, Inukai M. Studies on novel bacterial translocase I inhibitors, A-500359s. I. Taxonomy, fermentation, isolation, physico-chemical properties and structure elucidation of A500359 A, C, D and G. J Antibiot 56: 243-252 (2003)

2. Muramatsu Y, Ishii MM, Inukai M. Studies on novel bacterial translocase I inhibitors, A-500359s. II. Biological activities of A-500359 A, C, D and G. J Antibiot 56: 253-258 (2003)

3. Matthews RG. Cobalamin-dependent methyltransferases. Acc Chem Res 34: 681-689 (2001)

4. Pfohl-Leszkowicz A, Keith G, Dirheimer G. Effect of cobalamin derivatives on in vitro enzymatic DNA methylation: methylcobalamin can act as a methyl donor. Biochemistry 30: 8045-8051 (1991)

5. Yamaguchi H, Sato S, Yoshida S, Takada K, Itoh M, Seto H, Otake N. Capuramycin, a new nucleoside antibiotic. Taxonomy, fermentation, isolation and characterization. J Antibiot 39: 1047-1053 (1986) 